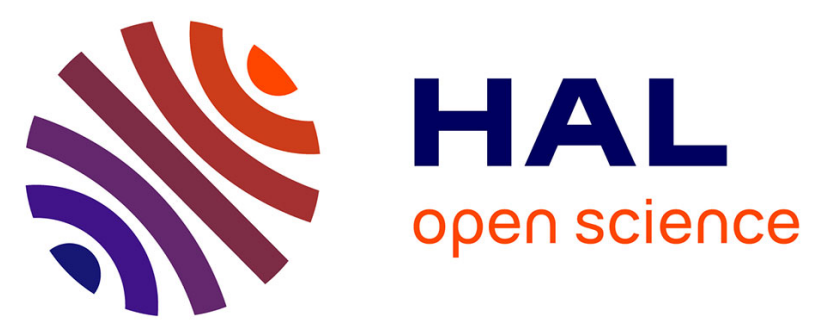

\title{
Ultrasound-triggered delivery of paclitaxel encapsulated in an emulsion at low acoustic pressures
}

\author{
N. Al Rifai, S. Desgranges, D. Le Guillou-Buffello, A. Giron, W. Urbach, M.
} Nassereddine, J. Charara, C. Contino-Pépin, N. Taulier

\section{- To cite this version:}

N. Al Rifai, S. Desgranges, D. Le Guillou-Buffello, A. Giron, W. Urbach, et al.. Ultrasound-triggered delivery of paclitaxel encapsulated in an emulsion at low acoustic pressures. Journal of materials chemistry B, In press, 10.1039/C9TB02493J . hal-02468818

\section{HAL Id: hal-02468818 \\ https://hal.sorbonne-universite.fr/hal-02468818}

Submitted on 6 Feb 2020

HAL is a multi-disciplinary open access archive for the deposit and dissemination of scientific research documents, whether they are published or not. The documents may come from teaching and research institutions in France or abroad, or from public or private research centers.
L'archive ouverte pluridisciplinaire HAL, est destinée au dépôt et à la diffusion de documents scientifiques de niveau recherche, publiés ou non, émanant des établissements d'enseignement et de recherche français ou étrangers, des laboratoires publics ou privés. 


\title{
Journal Name
}

\section{ARTICLE TYPE}

Cite this: DOI: $00.0000 / x x x x x x x x x x$

\section{Ultrasound-triggered delivery of paclitaxel encapsu- lated in emulsion at low acoustic pressures ${ }^{\dagger}$}

\author{
N. Al Rifai, ${ }^{a, b}$ S. Desgranges, ${ }^{c}$ D. Le Guillou-Buffello, ${ }^{a}$ A. Giron, ${ }^{a}$ W. Urbach, ${ }^{a, d}$ M. \\ Nassereddine,${ }^{b}$ J. Charara,${ }^{b}$ C. Contino-Pépin, ${ }^{c}$ and N. Taulier ${ }^{* a}$
}

Received Date

Accepted Date

DOI: 00.0000/xxxxxxxxxx

\begin{abstract}
We investigated the in vitro ultrasound-triggered delivery of paclitaxel, a well known anticancerous drug, encapsulated in an emulsion and in the presence of CT26 tumor cells. The emulsion was made of nanodroplets, which volume comprised $95 \%$ of perfluoro-octyl bromide and $5 \%$ of tributyl O-acetylcitrate, where paclitaxel was solubilized. These nanodroplets, prepared using a high-pressure microfluidizer, were stabilized by a tailor-made and recently patented biocompatible fluorinated surfactant. The delivery investigations were performed at $37^{\circ} \mathrm{C}$ using a high intensity focused ultrasound transducer at a frequency of $1.1 \mathrm{MHz}$. The ultrasonic pulse was made of 275 sinusoidal periods, the pulse repetition frequency was $200 \mathrm{~Hz}$ with a duty cycle of $5 \%$. The measured viabilities of CT26 cells showed that paclitaxel delivery was achievable for peak-to-peak pressures of 0.4 and $3.5 \mathrm{MPa}$, without having to vaporize the perfluorocarbon part of the droplet or to induce inertial cavitation.
\end{abstract}

\section{Introduction}

Treatment of cancer can engage severe treatments involving radiation and chemicals for several months or years that lead to undesirable side effects ${ }^{1}$. When considering drugs, the treatment can be efficient only if the drug concentration at the tumor site is over a critical concentration. Since a drug is not selective, it will be distributed in the whole patient body, which explains the dangerousness of most anti-cancerous drugs (such as paclitaxel or doxorubicin) that act indifferently on normal and tumoral tissues ${ }^{23}$. A way to solve this hurdle is to develop drug carriers in order to deliver most of the anti-cancerous drug at the tumor site, thus decreasing the drug concentration in the rest of the patient body, while protecting the drug from degradation 4 . Such drug carriers are usually injected intravenously and can accumulate at the tumor site either by passive targeting using the enhanced permeation and retention (EPR) effect $\frac{5[6]}{6}$ or by targeting the carrier to specific receptors of tumor tissues ${ }^{4}$. This approach improves the drug efficiency while decreasing side effects

\footnotetext{
a Sorbonne Université, CNRS, INSERM, Laboratoire d'Imagerie Biomédicale, LIB, F75006 Paris, France.

${ }^{b}$ Faculté des Sciences, Université Libanaise, Liban.

c Équipe Chimie Bioorganique et Systèmes Amphiphiles, Institut des Biomolécules Max Mousseron, UMR 5247, Université d'Avignon, Avignon, France.

${ }^{d}$ Laboratoire de Physique de l'École normale supérieure, ENS, Université PSL, CNRS, Sorbonne Université, Université de Paris, F-75005 Paris, France.

*E-mail: nicolas.taulier@sorbonne-universite.fr

$\dagger$ Electronic Supplementary Information (ESI) available: [details of any supplementary information available should be included here]. See DOI: 00.0000/00000000.
}

and patients' pain 6 . The efficiency can be further improved by controlling the drug release, using carriers sensitive either to the characteristics of the tumor tissue $(\mathrm{pH}$, temperature or redox potential) 9 or to an external stimulus (light, cold plasma, radiowave, magnetism, ultrasound... 10 . The use of ultrasound as a stimulus is appealing as it is easy to use and affordable. Ultrasound can also be used to image the localization of drug delivery or can be combined with other imaging modalities such as magnetic resonance imaging (MRI). More importantly, ultrasound can be easily focused on a small area of interest which brings new therapeutics applications 11 . High intensity focused ultrasound (HIFU) is currently being used in clinics for tumor thermal ablation 12 for which commercial apparatus (from EDAP TMS, Insightec or Chongqing Haifu Medical Technology Co. Ltd) has been specifically developed 11 . Such apparatus can be readily used in ultrasound-triggered drug delivery 115 . But this last application is still in the stage of preclinical research due to the lack of clinically approved sonosensitive drug carriers, even if some are in clinical trial such as Thermodox ${ }^{\circledR 16}$.

Paclitaxel (PTX) is a potent anticancer agent active against a broad spectrum of cancers and extensively used in cancer chemotherapy 17 . At high concentration, paclitaxel acts by inducing mitotic arrest while at low concentration, such that used in clinics, it may rather induce a multipolar division, as reported by Weaver 18 . Due to its low solubility in water, paclitaxel is clinically used either solubilized in Cremophor EL (Taxol ${ }^{\circledR}$ ) or bound to albumin (Abraxane ${ }^{\circledR}$ ). The second one exhibits less severe side effects than the first one ${ }^{19}$. However, there is still a need 
to decrease side effects as well as improving paclitaxel efficiency. Several types of sonosensitive and paclitaxel-loaded carriers have been investigated in the literature. A first type are free microbbubles 2021 or microbbubles encapsulated into liposomes 22 . These carriers exhibit a short life time and can not take advantage of the EPR effect due to their micrometric size. The paclitaxel delivery is triggered by the bubble stable cavitation generated by the ultrasound wave and this delivery is increased when inertial cavitation occurs. The second type are emulsions containing perfluoropentane (PFP), for which delivery is induced by the ultrasoundtriggered vaporization of PFP droplets 2324 . Rapoport et al. also induced paclitaxel delivery by replacing PFP with perfluoro-15crown-5-ether (PCFE), but higher pressures were needed (peak negative pressure, PNP, varying from 2.4 to $4.8 \mathrm{MPa}$ ) that led in their in vivo experiments to the death of several mice. While for $\mathrm{PFP}$, the peak negative pressure was no more than $1 \mathrm{MPa} 23 \mid 24$. A last type is made of micelles of mPEG-PLA-tocopherol ${ }^{25}$ for which paclitaxel delivery was achieved in the absence of cavitation, but micelles cannot load large paclitaxel quantity.

In this article, we investigated the possibility to trigger paclitaxel delivery by applying low acoustic pressures, for which ultrasound did not induce inertial cavitation, droplet vaporization or/and important temperature elevation. For this purpose, we used an emulsion as drug carrier, made of nanodroplets stabilized by a new class of recently patented fluorinated surfactant with a liquid core partitioned in a perfluorocarbon liquid part and an oily solubilizing paclitaxel.

\section{Materials and Methods}

\subsection{Materials}

Perfluorooctyl bromide (PFOB) were purchased from ABCR $\mathrm{GmbH}$ (Germany). Tributyl O-acetylcitrate or acetyl tributyl citrate (ATBC), acetonitrile, and methanol were purchased from Sigma-Aldrich Chemical (Saint-Quentin Fallavier, France). Paclitaxel was obtained from Chemieliva Pharmaceutical Co., Ltd. Dulbecoo's minimal essential serum, fetal bovine serum and penicillin streptomycin, trypsin (1X) and trypan blue were purchased from ThermoFisher Scientific (Gibco, France). The CT26-murine colorectal carcinoma cell line was purchased from ATCC. Fluorinated surfactants, $\mathrm{F}_{n} \mathrm{~d}_{i} \mathrm{TAC}_{m}$, possess a fluorinated chain length of $n=6$ carbons with a double polar chain $(i=2)$ made of a repetition of $m=12$ tris(hydroxymethyl)aminomethane (TAC) water soluble units. $\mathrm{F}_{6} \mathrm{~d}_{2} \mathrm{TAC}_{12}$ is a member of a recently developed new class of biocompatible branched surfactants called "DendriTAC"26. Their convergent synthesis allows stepwise modifications of the surfactant's size, shape, hydrophilic/lipophilic balance, nature of the head, number of tails, spacers or connecting units between hydrophilic head and hydrophobic tail. Their highly versatile structure affords not only to control the formation of structurally well-defined macromolecules but also to adjust step-bystep their self-assembling properties.

\subsection{Emulsion preparation}

About $7 \mathrm{mM}$ of $\mathrm{F}_{6} \mathrm{~d}_{2} \mathrm{TAC}_{12}$ surfactants were dissolved in a $15 \mathrm{~mL}$ centrifuge plastic tube containing $4 \mathrm{~mL}$ of $0.9 \%$ sodium chlo- ride solution. Thus, the surfactant concentration was higher than $0.056 \mathrm{mM}$, its critical micellar concentration (CMC). $0.57 \mathrm{~mL}$ of PFOB and then $0.03 \mathrm{~mL}$ of tributyl O-acetylcitrate (so that ATBC represents $5 \%$ of the droplet volume and PFOB 95\%) were added and the whole solution was mixed using a vortex to form a coarse emulsion.

For paclitaxel-loaded emulsions, paclitaxel has to be solubilized into tributyl $\mathrm{O}$-acetylcitrate before producing the coarse emulsion. In our experiments, the concentration of paclitaxel in ATBC varied from 105 to $200 \mu \mathrm{g} / \mathrm{mL}$, which is the maximum concentration we could achieve, but the first concentration was used on most experiments and in particular with CT26 cells.

The coarse emulsion was further emulsified using a high pressure homogenizer (model LV1 from Microfluidics): 8 passages were performed at a pressure of $20,000 \mathrm{psi}(\approx 138 \mathrm{MPa})$. The resulting emulsion was centrifuged at $6,000 \mathrm{rpm}$ (i.e. at a relative centrifugal force of 2,000 g) for $30 \mathrm{~s}$ with a bench mini-centrifuge (Mini Star from VWR) to force any microdroplets to sediment. The surpernatant only contained nanodroplets and possibly free paclitaxel.

When paclitaxel was present, the supernantant was further centrifuged, this time at 13,300 rpm (i.e. at 17,000 g) for $20 \mathrm{~min}$ using a bench centrifuge (micro Star $17 \mathrm{R}$ from VWR) so that most nanodroplets formed a pellet. The supernatant was removed and replaced with a fresh $0.9 \%$ sodium chloride solution. A vigorous mixing led to the resuspension of the nanodroplets in a solution free of paclitaxel.

The droplet concentration in the emulsion is approximatively of $(61 \pm 1) \times 10^{11}$ droplets per $\mathrm{mL}$, which corresponds to a droplet volume fraction of approximatively $5.8 \%$. The emulsion was eventually stored into the fridge at $4^{\circ} \mathrm{C}$ to a maximum of three days during which parts of the solution was used to perform experiments.

\subsection{Droplet size measurements}

The mean diameter of the droplets forming the emulsion was measured by dynamic light scattering using a ALV/CGS-3 platform based goniometer system (from ALV GmbH). The measurements were performed on emulsions diluted 100 times at room temperature and at several scattering angles from $50^{\circ}$ to $130^{\circ}$, with a step of $20^{\circ}$. At each angle $\theta$, the device provided the decay rate $\Gamma_{\theta}$ whose values were plotted as a function of the scattering vector amplitude $q(\theta)=\frac{4 \pi n}{\lambda} \sin (\theta / 2)$, where $n=1.333$ is the refractive index of the solution and $\lambda=633 \mathrm{~nm}$ is the laser wavelength. A fit of the curve by the cumulant method allowed to determine the droplet mean diameter $D$ along with the polydispersity index (PDI) 27 .

\subsection{Determination of the amount of encapsulated paclitaxel}

At the end of the emulsion preparation, one of the centrifuged tube, which contained the pellet of a $1 \mathrm{~mL}$ centrifuged emulsion, was used to assess the quantity of encapsulated palictaxel. The supernatant obtained after the second centrifugation was replaced by $25 \mu \mathrm{L}$ of fresh $0.9 \%$ sodium chloride solution and $975 \mu \mathrm{L}$ of solution of acetonitrile, methanol and water (3:3:1 
$(\mathrm{v} / \mathrm{v}))$. The whole sample was then vortexed to extract and solubilize paclitaxel that was encapsulated into the nanodroplets forming the pellet. A $1 \mathrm{~cm}$ pathlength quartz cuvette (from Hellma) was filled with this solution and its absorbance was recorded by a V-730 UV-Visible spectrophotometer (from Jasco) at a wavelength of $227 \mathrm{~nm}$ and at $25^{\circ} \mathrm{C}$. The concentration, $C$, of paclitaxel was obtained from the absorption value, thanks to a calibration curve previously determined from paclitaxel dissolved in acetonitrile/methanol/water (3:3:1 (v/v)) in the concentration range of $1-20 \mu \mathrm{g} / \mathrm{mL}$.

\subsection{HIFU system}

Fig. 1 shows an illustration of the ultrasonic setup used to trigger the release of paclitaxel or Nile red encapsulated into the emulsion. In this setup, a waveform generator (model 33220A from Agilent) generates an electrical signal that goes first through a radio-frequency power amplifier (Model 150A100C from AR France), then a power reflection meter (Model \& NRT from Rohde Schwarz) measures the delivered average electrical power. The electrical signal is converted into an acoustic wave by a focused transducer (Model H-101-G from Sonic concepts Inc.), whose fundamental frequency mode is at $1.1 \mathrm{MHz}$. The acoustic wave propagates into a water tank, which is degassed and thermostated at $37 \pm 0.2^{\circ} \mathrm{C}$ using a degassing machine (Model WDS-1005 from Sonic Concepts) connected to the tank. A tube, completely filled with $250 \mu \mathrm{L}$ of sample, is placed at the transducer focus. A focused hydrophone (model Y-107 from Sonic Concepts), whose focus overlaps the transducer focus inside the tube, continuously monitors the signal emitted by the sample and is used to detect cavitation. The overlapped focus volume had been optimized by maximalizing the acoustic amplitude of the fundamental pic (at $1.1 \mathrm{MHz}$ ) measured by the hydrophone when the transducer emits a low amplitude signal (i.e. in the absence of cavitation). The position of the tube was optimized by filling the tube with a viscous solution containing microbubbles and maximalizing the amplitude of the recorded harmonic signal (at $2.2 \mathrm{MHz}$ ) emitted by the insonified bubbles (in the stable cavitation regime). Calibration measurements were performed with a fiber optic hydrophone (from Precision Acoustic) placed inside the tube (containing water) at the transducer focus to measure the peak-topeak acoustic pressure $P_{p k p k}$ and temperature elevation $\Delta T$ due to insonification. These values were correlated to the average power measured by the wattmeter. The fiber optic hydrophone was positioned so that the recorded amplitude of the ultrasonic signal emitted by the transducer was maximalized in the absence of cavitation. In our experiment, the signal consisted of sine-wave bursts at a frequency $(f)$ of $1.1 \mathrm{MHz}$, with a duty cycle $(D C)$ of $5 \%$ and a pulse repetition frequency $(P R F)$ of $200 \mathrm{~Hz}$. The time of insonification $\tau$ was either $3,6,12$ or $20 \mathrm{~min}$.

\subsection{Terephthalate dosimeter}

The presence of inertial cavitation was assessed using a terephatalate dosimeter according to the procedure described by Somaglino et al.28. The HTA dosimetry is sensitive enough when insonification time exceeds a minute ${ }^{299}$. For an insonification last-

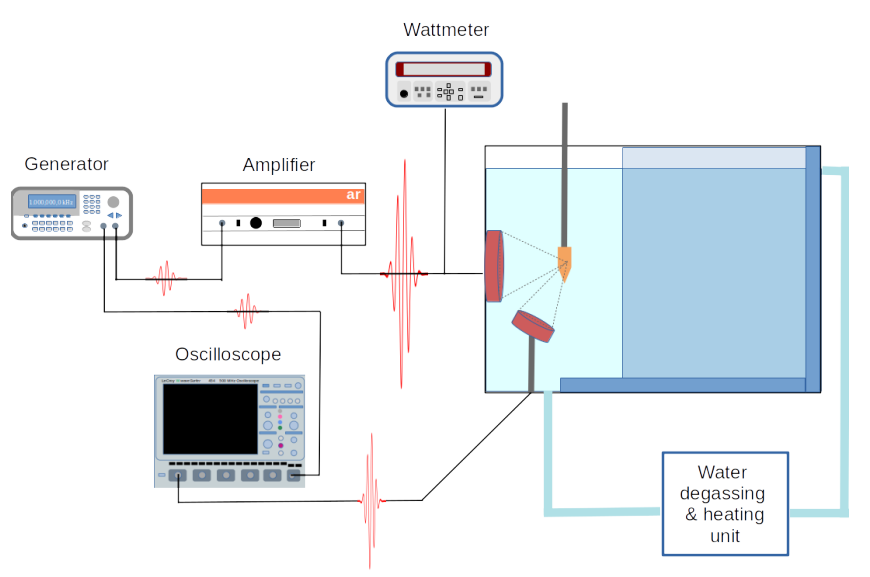

Fig. 1 Illustration of the ultrasonic setup used to insonify the samples. See text for a detailed description.

ing more than a minute as it is the case in our experiments, Somaglino et al. showed the equivalence between HTA and ultrasonic dosimetry to evaluate the cavitation dose $\mathrm{e}^{28}$. The principle consists in measuring the quantity of hydroxyl radicals $\left(\mathrm{OH}^{\bullet}\right)$ produced by inertial cavitation. This quantity was obtained by measuring the increase in fluorescence intensity due to the presence of fluorescent 2-hydroxylterephthalate (HTA), which is produced by the binding of non-fluorescent terephthalate (TA) to hydroxyl radicals.

Fluorescence spectra were measured using a spectrophotometer (model FP 8300 from Jasco) at an excitation wavelength of $318 \mathrm{~nm}$ and at an emission wavelength of $426 \mathrm{~nm}$. First, a reference spectrum was obtained by averaging spectra measured on several solutions at a HTA concentration of $1 \mu \mathrm{M}$. One concentration was enough since HTA fluorescence is linearly proportional to HTA concentration in the range $0.2-20 \mu \mathrm{M}, 30 \mid 31$ and HTA concentration produced by inertial cavitation was always lower than $20 \mu \mathrm{M}$ in our samples. Next, the spectra of samples containing $2 \mathrm{mM}$ TA were measured before and after insonification for a desired set of ultrasonic parameters. The concentration of HTA was derived using the following equation:

$$
C_{\mathrm{HTA}}=\frac{F_{\mathrm{after}}-F_{\text {before }}}{F_{\mathrm{ref}}} \times C_{\text {ref }}
$$

where $C_{\text {ref }}$ is the HTA concentration in the reference solution (i.e. $1 \mu \mathrm{M}), F_{\text {ref }}$ is the fluorescence spectra of this reference solution, $F_{\text {before }}$ and $F_{\text {after }}$ are the spectra of the solution containing initially $2 \mathrm{mM}$ TA, respectively, before and after insonification.

\subsection{Release experiments using encapsulated Nile red}

The evaluation of the ultrasound triggered release efficiency was evaluated from droplets encapsulating Nile red. The droplet production followed the protocol used to encapsulate paclitaxel except that the second centrifugation was not performed. Consequently, the emulsion contained free Nile red in addition to encapsulated Nile red. Next, $150 \mu \mathrm{L}$ of emulsion was added to the tube to be insonified or incubated (i.e. with no insonification) and the rest of the tube volume (i.e. $100 \mu \mathrm{L}$ ) was filled with ATBC, in 
this configuration free Nile red migrates from the emulsion to the ATBC supernatant. After an insonification or incubation time $\tau$, a fraction of the ATBC supernatant was taken out and its fluorescence intensity was measured $\left(\lambda_{e x}=530 \mathrm{~nm}\right.$ and $\left.\lambda_{e m}=560 \mathrm{~nm}\right)$. Using a calibration curve relating Nile red intensity to its concentration, we evaluated the concentration of Nile red, $C_{\mathrm{RN}}$, located in the ATBC supernatant.

\subsection{Experiments using tumor cells}

The CT26-murine colorectal carcinoma cell line was maintained at $37^{\circ} \mathrm{C}$ in DMEM (Dulbeco's modified eagle's medium, high glucose, GlutaMAX supplement) supplemented with 10\% of heat-inactivated fetal bovine serum (FBS) and 1\% of penicillin/streptomycin. Cells were cultured in a $\mathrm{CO}_{2}$ incubator (model MCO-18AC-PE from panasonic) and were passaged twice a week by removing the adherent cells with trypsin/EDTA. Before any experiment, CT-26 cells were seeded for $48 \mathrm{~h}$ into T75 flasks in DMEM supplemented with 10\% FBS and 1\% penicillin/strepromycin. Solutions of CT-26 cells from passages 2:5 were used for experiments. For experiments involving nanodroplets, the solution was centrifuged after insonification at $1000 \mathrm{rpm}$ (i.e. $96 \mathrm{~g}$ ), $5 \mathrm{~min}$, and room temperature. The supernatant was removed to get rid of nanodroplets and of free paclitaxel. The pellet was immediately resuspended in DMEM supplemented with 10\% FBS and 1\% penicillin/strepromycin. Following every experiment, solutions were being seeded in 24 well plates (with $10^{5}$ cells/well). Some plates were incubated for $24 \mathrm{~h}$ while others for $48 \mathrm{~h}$. After incubation, the number of living and dead cells were counted using an automatic cell counter (model EVE from VWR) and trypan blue assay. The cell viability was assessed by calculating the ratio of the living cell number to the total number of cells (i.e. living + dead cells).

\subsection{Statistical analysis}

The experimental design is a full factorial design with four factors (drug, incubation duration, pressure value, insonification duration) and repeated measures $(n=3)$. Means and standard error of viability are displayed into Fig. 5. All statistical analyses were performed using the JMP software (SAS Institute Inc., Cary, NC, USA). The statistical level of significance was set to $p=0.05$. Scores were reported as means and standard deviation. Comparisons were performed with Anova and non-parametric Wilkoxon or Mann-Whitney tests when necessary. Fisher or Levene tests were used to compare variances. Multivariate analysis (Anova or Ancova) were followed by Tuckey-Kramer or non parametric Steel-Dwass-Critchlow-Fligner post-hoc tests (with controls) when necessary. Decomposition of variance was processed using Bayesian method.

\subsection{Figure preparation}

All figures were prepared using python 3.7 and matplolib 3.1.1. The fits in Fig. 3 and 4 were performed using the least square function provided by scipy 1.3.0.

\begin{tabular}{ccc}
\hline $\begin{array}{c}\mathrm{D} \\
(\mathrm{nm})\end{array}$ & PDI & $\begin{array}{c}C \\
(\mu \mathrm{g} / \mathrm{mL})\end{array}$ \\
\hline $273 \pm 6$ & 0.10 & 0 \\
$272 \pm 6$ & 0.26 & 3.2 \\
$221 \pm 4$ & 0.19 & 4.1 \\
$258 \pm 6$ & 0.39 & 44 \\
\hline
\end{tabular}

Table 1 The first and second columns display the droplet diameter and polydispersity as given by light scattering technique, and the third column gives the concentration of encapsulated paclitaxel $(C)$ in the emulsion.

\section{Results}

\subsection{Properties of the emulsion}

The droplet diameter decreased for increasing amount of surfactant added during the emulsion preparation. We chose to work with a diameter of approximatively $270 \mathrm{~nm}$ to investigate drug delivery, but the lower achievable diameter was $70 \mathrm{~nm}$. The droplet diameter further depended on the quantity of encapsulated paclitaxel (see Table 1). For low paclitaxel concentrations $C$ (several micrograms per milliliter), the droplet size was reduced when the quantity of paclitaxel increased, which was not true when reaching a larger concentration (i.e. at $C=44 \mu \mathrm{g} / \mathrm{mL}$ ). For comparison purpose, we used a paclitaxel concentration of $3.2 \mu \mathrm{g} / \mathrm{mL}$ so that free and drug-loaded droplets exhibited the same diameter. All emulsion formulations exhibited a polydispersity index (PDI) value below 0.4 , regardless of paclitaxel concentration, knowing that a value smaller than 0.2 is characteristic of a monodispersity in droplet size. The amount of paclitaxel encapsulated into the emulsion represents 3 to $9 \%$ of the quantity of paclitaxel added during the emulsion preparation. This indicates that most paclitaxel had been lost during the emulsion preparation, due to both centrifugations.

Finally, we investigated the effect of nanodroplets devoid of paclitaxel on the toxicity of CT-26 cells, where an increasing volume fraction (i.e. $0.1,1,5$ and $10 \%$ ) is made of the emulsion instead of DMEM supplemented with 10\% FBS and 1\% penicillin/strepromycin. This corresponds to a droplet volume fraction $\phi=0.0058,0.058,0.116$ and $0.58 \%$, respectively. Fig. 2 shows that CT26 viability is weakly affected for $\phi=0.0058 \%$ and it dramatically decreases for 0.116 and $0.58 \%$. Consequently, we chose to work with the safer condition, that is $\phi=0.0058 \%$, in experiments dealing with CT26 cells, which corresponds to a ratio of 60 nanodroplets for one CT26 cell.

\subsection{Characteristic of the ultrasound}

We used an ultrasound wave where only the acoustic pressure and the time of insonification were varied. The other ultrasonic parameters, that were kept constant, were the frequency $(f=1.1 \mathrm{MHz})$, the duty cycle $(D C=5 \%)$ and the pulse repetition frequency $(P R F=200 \mathrm{~Hz})$. Using a fiber optic hydrophone $(\mathrm{FOH})$, we measured a temperature elevation of 1 and $4^{\circ} \mathrm{C}$ for peak-to-peak pressures $P_{p k p k}$ of 0.4 and $3.5 \mathrm{MPa}$, respectively. There was no inertial cavitation detected at these two pressures as shown by the result from the terephalate dosimeter (Fig. 3) and by the lack of characteristic signal recorded by the focused 


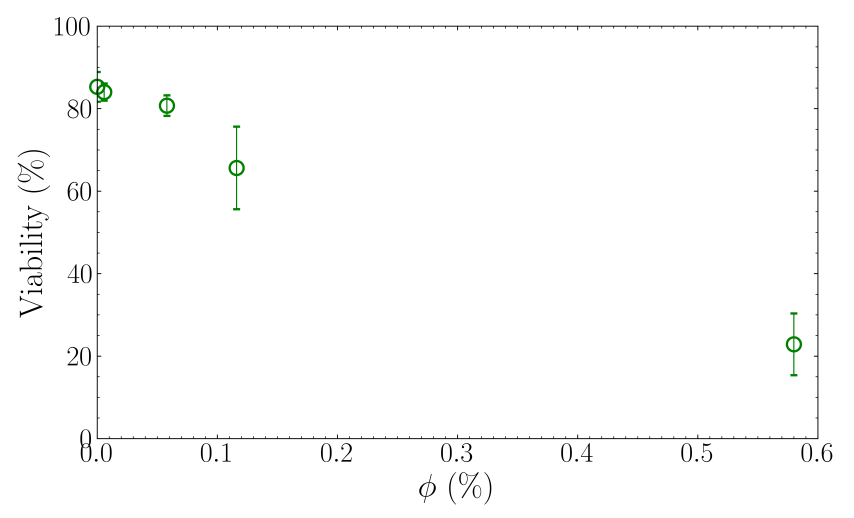

Fig. 2 Variation in the viability of CT26 cells in the presence of an increasing volume fraction $\phi$ of nanodroplets devoid of paclitaxel.

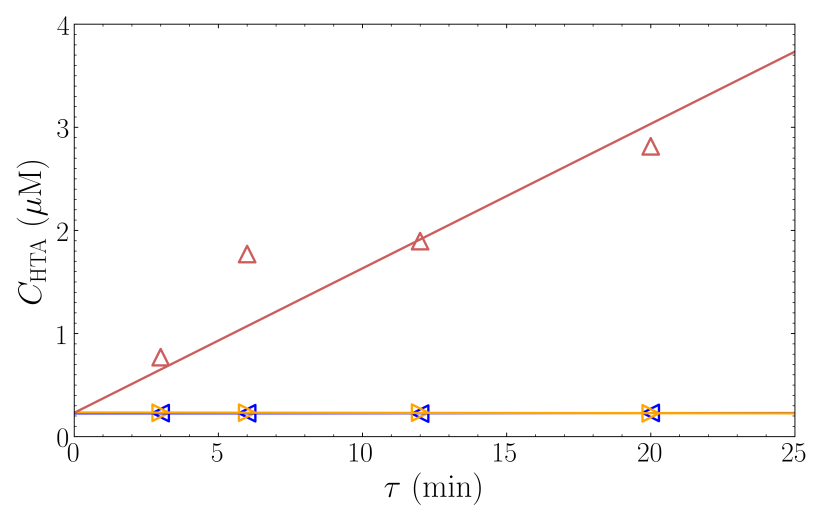

Fig. 3 Variation in the concentration of 2-hydroxyl terephtalate (THA) as a function of insonification time $\tau$ for acoustic peak-to-peak pressures of $0.4 \mathrm{MPa}(\triangleleft), 3.5 \mathrm{MPa}(\triangleright)$, and $7 \mathrm{MPa}(\triangle)$, at $37^{\circ} \mathrm{C}$ and using $D C=5 \%$ and $P R F=200 \mathrm{~Hz}$.

hydrophone. This hydrophone also did not detect stable cavitation at these pressures. Note that the ultrasonic pulse comprises 275 sinusoidal periods that was enough to detect an harmonic response from bubble stable cavitation. These results were identical whatever the insonification time.

Whereas, experiments made at a higher pressure of $7 \mathrm{MPa}$ clearly showed the presence of inertial cavitation as shown in Fig. 3. with a cavitation dose increasing with the insonification time. The presence of cavitation was also detected by the focused hydrophone which recorded a signal made of harmonics and of a broadband frequency noise. A temperature elevation of $10^{\circ} \mathrm{C}$ was measured.

\subsection{Effect of pressure in drug delivery}

We evaluated in Fig. 4 the variation in Nile red concentration inside the ATBC solution located on top of the emulsion. In the absence of ultrasound, the detection of Nile red reflected the fact that the emulsion contained a substantial quantity of free Nile red as we did not get rid of them. However, the Nile red concentration in the ATBC solution did not vary with the incubation time. At ultrasonic pressures of 0.4 and $3.5 \mathrm{MPa}$, the Nile red concentration increased linearly with insonification time. For data corresponding to $7 \mathrm{MPa}$, an heuristic curve (i.e. $f(\tau)=a+b \times \frac{\tau}{c+\tau}$ ) was

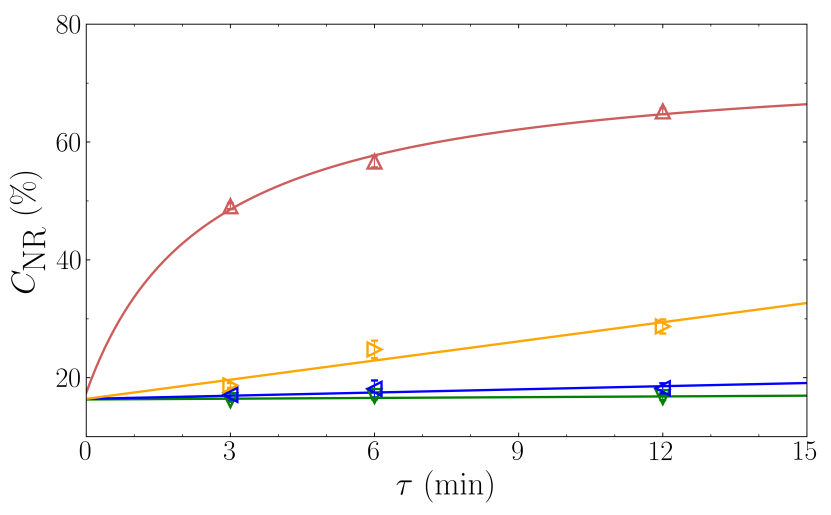

Fig. 4 Percentage of Nile red measured on a ATBC solution located on top of an emulsion when incubated $(\nabla)$, i.e. non-insonified, or insonified at a pressure of $0.4,3.5$ or $7 \mathrm{MPa}(\triangleleft, \triangleright$ and $\triangle$ respectively) during a time $\tau$ of 3,6 , or $12 \mathrm{~min}$. For $P_{p k p k}<7 \mathrm{MPa}$, a linear fit led to a slope of $0.04,0.20$, and 1.11 for $0,0.4$ and $3.5 \mathrm{MPa}$, respectively. All fit curves start at approximatively $16.6 \%$ at $t=0$.

used instead. In Fig. 4, all fits start at $\tau=0$ at the concentration derived from the fit of incubation experiments (green line).

\subsection{Experiments on CT26 cells}

In these experiments, cell samples were either insonified or incubated (i.e. with no ultrasound) during a time $\tau=3,6,12$ or $20 \mathrm{~min}$ and the cell viabilities were measured 24 or $48 \mathrm{~h}$ later.

In a first series of control experiments (top figure in Fig [5), the viability was determined for cell solutions insonified or incubated in the absence of emulsion. The application of ultrasound (at 0.4 or 3.5 MPa, blue and orange symbols, respectively) induced a small reduction in cell viability as compared to non-insonified solutions (gray symbols).

In a second series of control measurements (middle figure in Fig 5), the viability of CT26 cells was measured in the presence of nanodroplets devoid of paclitaxel at a volume fraction of $0.0058 \%$. In the absence of ultrasound, the cell viability was sligthly affected by the presence of nanodroplets (green symbols) compared to the control sample (with no nanodroplets or ultrasound, gray symbols). While the cell viability weakly decreased, but still remained over $90 \%$, when insonified at 0.4 or $3.5 \mathrm{MPa}$ (blue and orange symbols, respectively).

In the last series of experiments (bottom figure in Fig. 5), measurements were performed in the presence of nanodroplets encapsulating paclitaxel. The droplet volume fraction and the droplet size were identical to the second series of control experiments, that is $\phi=0.0058 \%$ and $270 \mathrm{~nm}$, respectively. This gave a concentration of $3.2 \mu \mathrm{g} / \mathrm{mL}$ of encapsulated paclitaxel into the cell solution. In the absence of ultrasound, the presence of paclitaxel-loaded nanodroplets reduced the cell viability (green symbols) by no more than $10-15 \%$ (with a viability always over $70 \%$ ), 24 or $48 \mathrm{~h}$ after incubation compared to control solutions (i.e. without insonification or nanodroplets, gray symbols). The viability was drastically reduced in the presence of ultrasound (blue and orange symbols) with values going down below $50 \%$ at $\tau=20 \mathrm{~min}$. The cell viability linearly decreased with the insonifi- 

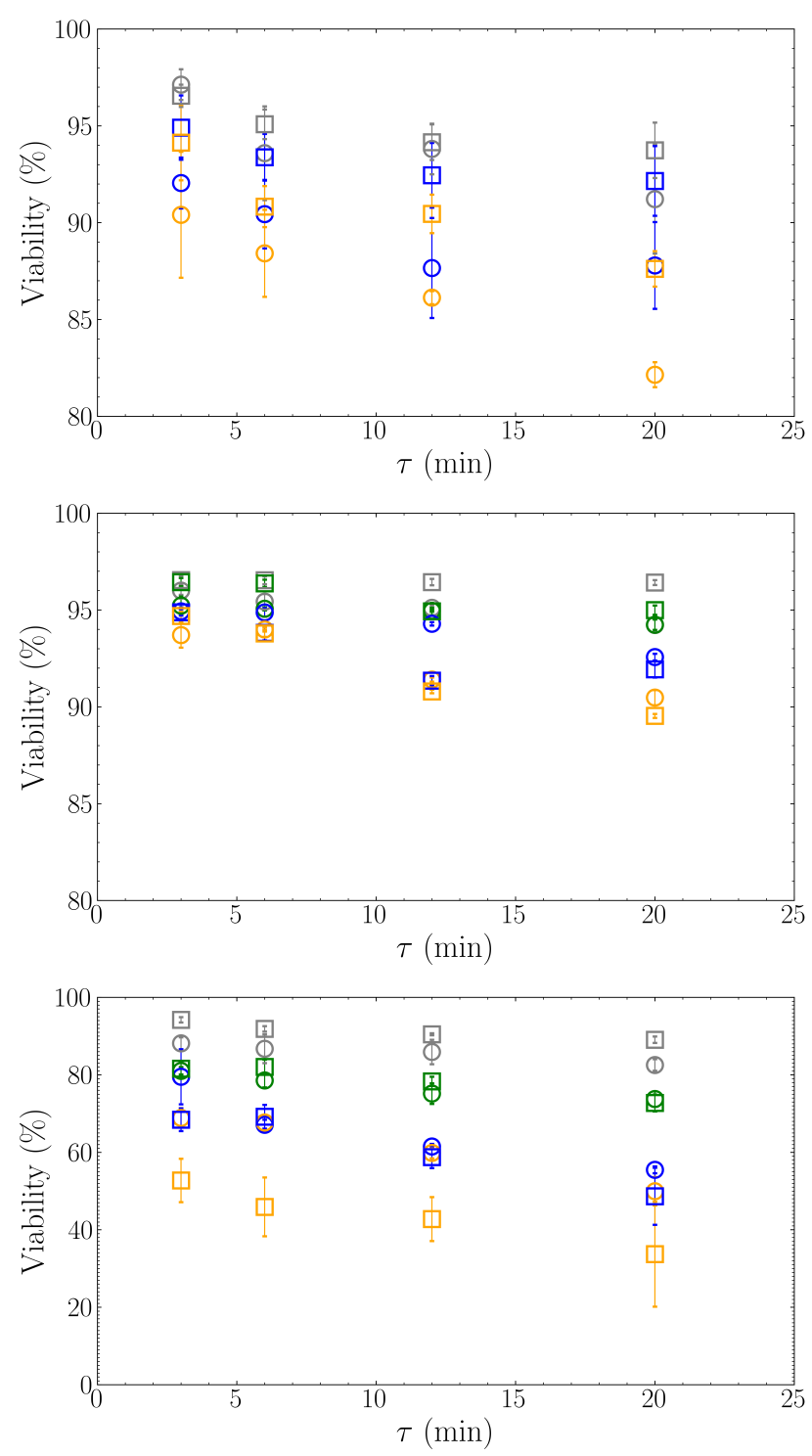

Fig. 5 Viabilities of CT26 cells measured at $24 \mathrm{~h}$ (circles) and $48 \mathrm{~h}$ (squares) after being insonified or incubated during a time $\tau$ at $37^{\circ} \mathrm{C}$ in the absence of emulsion (top figure), in the presence of emulsion ( $\phi=0.0058 \%$ ) devoid of paclitaxel (middle figure), in the presence of emulsion ( $\phi=0.0058 \%$ ) encapsulating paclitaxel (bottom figure). The gray symbol ( $\bigcirc$ and $\square$ ) are for solutions of CT26 cells incubated during a time $\tau$ (without ultrasound or emulsion), for CT26 solutions incubated during $\tau$ in the presence of emulsion without ultrasound ( $\bigcirc$ and $\square$ ) or insonified during $\tau$ at a pressure of $0.4 \mathrm{MPa}(\bigcirc$ and $\square$ ) or $3.5 \mathrm{MPa}(\bigcirc$ and $\square$ ). cation time (with $p<10^{-3}$ ) with no large difference between 24 or $48 \mathrm{~h}$ at $0.4 \mathrm{MPa}$, which was not the case at $3.5 \mathrm{MPa}$ (orange square symbols).

\section{Discussion}

The purpose of having PFOB in our droplets was to offer a detection by ${ }^{19} \mathrm{~F}$ MRI. This characteristic was the object of previously published articles on similar PFOB droplets ${ }^{32 / 33}$. PFOB emulsion was approved by FDA (the Food and Drug Administration) in 1993 as a contrast agent under the name Imagent and was also used ${ }^{34}$ in small animal studies and some human trials. Since perfluorocarbon liquid such as PFOB cannot solubilize hydrophobic drugs such as paclitaxel, a small volume (5\%) of ATBC was added to the droplet volume to solubilize them. ATBC has been approved by FDA as a substance for use as a pharmaceutical excipient. It is mainly used in pharmaceutical coating of solid oral dosage forms, such as coated tablets or capsules, as well as plasticizers in cosmetic ${ }^{35}$. The pharmacokinetic properties of ATBC have been investigated by Kim et al. $\frac{36}{\text {, }}$ in particular, ATBC exhibits a rapid clearance in rat. We do not have information about the distribution of ATBC into the droplet. But a previous study using triacetin instead of ATBC showed that triacetin forms a corona between the PFOB core and the surfactant shell for micrometric droplets 32 . The droplets were stabilized by a recently developed and patented fluorinated surfactant ${ }^{26}$. These surfactants present the advantage that their polar head is easily functionalized with RGD peptides for instance to target the droplets toward tumor tissues $37 / 38$. A pegylation is also not needed as the TAC structure composing the surfactant polar head provides a sufficient protection against the immune system 38 . Our data showed that these nanodroplets, devoid of paclitaxel, exhibited a low toxicity at a small droplet volume fraction (i.e. $\phi=0.0058 \%$ in Fig. 2), which is the concentration we used for the rest of experiments dealing with CT26 cells (i.e. in Fig. 5). The reduction in CT26 cell viability observed in Fig. 2 for larger volume fraction can be due to the loss of available nutrient in addition to droplet toxicity. Indeed up to $10 \%$ of the nutrient volume (at $\phi=0.58 \%$ ) was replaced by the emulsion in these measurements.

The quantity of surfactant required to produce an emulsion depends on the droplet radius we wish to achieve. Indeed, when using optimized parameters, the device used to produce emulsion (a high pressure homogenizer in our case) will create droplets with an initial size $D_{0}$. Droplets will grow immediately, mainly by coalescence. The surfactant solubilized into the aqueous solution will progressively coat the droplet surface and droplets will continue to grow until their surface is saturated by surfactants. The droplet size is then stabilized to a value $D$. Thus, the desired droplet size $D$ can be tuned by modifying the concentration of surfactant in the aqueous solution. In our case, we used a quantity of surfactant that lead to a droplet diameter of approximatively $270 \mathrm{~nm}$, in the absence of paclitaxel. The fact that a large amount of encapsulated paclitaxel reduced the nanodroplet size, suggests that paclitaxel was partly located at the surfactant shell. These emulsions remain stable for months. During this time, the droplet size slowly grows due to Oswald ripening when kept at a constant volume fraction 32 . In our case the size barely change as 
the emulsion were kept for no more than a few days.

In the paclitaxel-delivery experiments, we used peak-to-peak pressures of $0.4 \mathrm{MPa}$ and $3.5 \mathrm{MPa}$ that correspond to peak negative pressures (PNP) of 0.2 and $1.75 \mathrm{MPa}$, respectively. These values have been chosen because in medical echograph, the mechanical index ${ }^{39} \mathrm{MI}=\mathrm{PNP} / \sqrt{f}$ is limited to 1.9 and a value smaller than 0.3 is recognized to induce no bioeffects? This translates in our case to $P_{p k p k}$ values of approximately 4 and $0.6 \mathrm{MPa}$, respectively (with the frequency equals to $f=1.1 \mathrm{MHz}$ ), which were respectively larger than the values 3.5 and $0.4 \mathrm{MPa}$ we have chosen. In our case, no inertial cavitation occured at $0.4(\mathrm{MI}=0.2)$ and at 3.5 $\mathrm{MPa}$ ( $\mathrm{MI}=1.7$ ), while inertial cavitation was present at $7 \mathrm{MPa}(\mathrm{MI}>1.9)$ as shown by Fig. 3. In addition, the signal recorded by the focused transducer contained no signal characteristic of stable cavitation due to the appearance of bubbles or to droplet vaporization.

In most investigations dealing with ultrasound triggered drug release, one of the three following mechanisms is used. The first mechanism uses the temperature elevation induced by ultrasound to stimulate temperature sensitive carriers such as thermosensitive liposomes $\frac{16}{16}$ or polymers 40 . In a second mechanism, ultrasound induces cavitation from gas dissolved into the medium or thanks to the presence of injected microbubbles. In the first case, the carriers are liposomes 4344 , polymeric nanoparticles 45 or emulsions ${ }^{4}$ while in the second case, they are either microbubble $20 \mid 21 \sqrt{46}$ or microbubbles encapsulated into liposomes 22 47. In the third mechanism, ultrasound induces the vaporization of perfluorocarbon droplets ${ }^{48}$ which requires carriers containing one 2 or several 49 perfluorocarbon droplets. In our case, we wished not to use any of these three mechanisms but another less common one which been observed by Oerlemans et al. 50 for nonthermosensitive liposomes or by Howard et al. for polymeric micelles $^{25}$. In this mechanism, the release is expected to be due to a higher permeabilization of the shell that is enhanced by the ultrasonic radiation force, leading to a faster diffusion of the encapsulated drug out of the carrier. To establish if ultrasound can release an hydrophobic molecule encapsulated in our nanodroplets using this mechanism, Nile red was used over paclitaxel as the latter is not fluorescent. In Fig. 4 a continuous increase in Nile red concentration in the upper ATBC solution as a function of $\tau$ reflects an ultrasound-triggered release of Nile red since a passive release (i.e. without insonification) is characterized by a constant Nile red concentration (green symbols). The absence of cavitation or droplet vaporization observed at 3.5 MPa (orange symbols) combined with the linear increase in red nile concentration suggests a diffusion mechanism with a diffusion rate given by the linear slope. The release rate decreases with lower acoustic pressure (and temperature elevation) as shown at $0.4 \mathrm{MPa}$ (for which $\Delta T=1^{\circ} \mathrm{C}$ ) as the slope of the fit is five times lower than at 3.5 $\mathrm{MPa}$ and became not significantly different from the curve corresponding to no insonification. The presence of cavitation clearly changes the release kinetics of the encapsulated Nile red (red symbols in Fig. 4). Even if cavitation greatly improves Nile red release and can offer advantageous effects (e.g. cell sonoporation 51 , opening of the brain-blood barrier 52 53, blood vessel damage $\mathrm{e}^{54}$ ) that may improve drug efficiency, it is still better to avoid it as the benefit for a patient has yet to be clinically demonstrated $55-57$.

For viability experiments performed with CT26 cells, there was no statistical difference between experiments performed 24 or $48 \mathrm{~h}$ after incubation or insonification. The larger contribution to the overall variance comes from the presence or absence of paclitaxel as emphasized by the difference in viability variations observed between the middle and bottom figures of Fig. 5 . Overall, the values of viability significantly depends (with $p<10^{-3}$ ) on the presence/absence of paclitaxel, ultrasound and droplets as well as on the insonification/incubation time.

The control measurements displayed in Fig. 5 indicates that the conditions (droplet concentration and ultrasonic parameters) used in this study weakly affect CT26 cell viability with a variation never exceeding $7 \%$. In the absence of ultrasound, the presence of paclitaxel-loaded emulsion reduces the viability by approximatively $10 \%$ due to the passive release of paclitaxel during the time of incubation. Indeed, both paclitaxel and ATBC have a small but non-negligible solubility in water, $0.4 \mu \mathrm{g} / \mathrm{mL}$ and $4.49 \mathrm{mg} / \mathrm{mL}$, respectively. So both compounds continuously diffuse out of the droplets until the solvent is saturated with PTX and ATBC, which can be the case in vitro but rarely in vivo.

The further decrease in cell viability observed in the presence of ultrasound (bottom figure of Fig. 5) confirms an ultrasound triggered delivery of paclitaxel. An ultrasonic pressure of $0.4 \mathrm{MPa}$, accompanying by a small induced temperature elevation $\left(\Delta T=1^{\circ} \mathrm{C}\right.$ ), is enough to induce paclitaxel delivery with an efficient effect of CT26 tumors. However, it is surprising that after $24 \mathrm{~h}$ there is no apparent difference due to pressure, while experiments performed with Nile red (Fig. 4) suggests that a higher release of paclitaxel is expected at $3.5 \mathrm{MPa}$ than at $0.4 \mathrm{MPa}$. In addition, the variation of viability was clearly different between 24 and $48 \mathrm{~h}$ at $3.5 \mathrm{MPa}$ and this was the only conditions exhibiting such an important difference between the two times. A definitive explanation of the differences observed between 0.4 MPa and 3.5 MPa will require further investigations. It may originate from a synergistic action of ultrasound and paclitaxel 59 . The difference could also have been enhanced due to the higher temperature elevation $\left(\Delta T=4^{\circ} \mathrm{C}\right)$ and radiation force at $3.5 \mathrm{MPa}$.

\section{Conclusion}

We investigated a paclitaxel carrier made of nanodroplets, partitioned in PFOB (for ${ }^{19} \mathrm{~F}$ MRI purpose) and in ATBC (to solubilize paclitaxel), stabilized by a recently patented biocompatible fluorinated surfactant. Our carriers allows a delivery using lower peakto-peak pressures, with peak negative pressures of $P N P=0.2$ and $1.75 \mathrm{MPa}$, corresponding to mechanical indexes (MI) of 0.2 and 1.7 , respectively. This means that our carriers are compatible with the use of clinical echographs and lies within safe ultrasonic standards. Because the insonification time should last several minutes, the approach is more suited to treat tumoral tissues where nanodroplets have accumulated due to active or passive targeting. The mechanism of delivery is not related to cavitation nor acoustic droplet vaporization but is probably due to an enhanced permeabilization of the nanodroplet shell induced by ultrasonic radiation force. 


\section{Acknowledgment}

This work was supported by "Plan cancer 2014-2019" (projects AIDA and BubDrop4Glio) and the Ph.D. scholarship of N. Al Rifai was funded by CNRS-L/UL.

\section{Notes and references}

1 A. McClure, J. Diagn. Med. Sonogr., 2016, 32, 343-350.

2 N. Y. Rapoport, A. M. Kennedy, J. E. Shea, C. L. Scaife and K.-H. Nam, J. Control. Rel., 2009, 138, 268-276.

3 K. H. Bae, H. J. Chung and T. G. Park, Mol. Cells, 2011, 31, 295-302.

4 G. P. Kumar and A. Divya, Med. Chem., 2015, 5, 1-13.

5 H. Maeda, J. Wu, T. Sawa, Y. Matsumura and K. Hori, J. Control. Rel., 2000, 65, 271-284.

6 F. Man, T. Lammers and R. T. M. de Rosales, Mol. Imaging Biol., 2018, 20, 683-695.

7 C. Riggio, E. Pagni, V. Raffa and A. Cuschieri, J. Nanomater., 2011, 2011, -.

8 T. Tagami and T. Ozeki, Journal of Pharmaceutical Sciences, 2017, 106, 2219 - 2226.

9 S. Ganta, H. Devalapally, A. Shahiwala and M. Amiji, J. Control. Release, 2008, 126, 187-204.

10 J. Yao, J. Feng and J. Chen, Asian J. Pharm. Sci., 2016, 11, 585-595.

11 Y.-S. Kim, H. Rhim, M. J. Choi, H. K. Lim and D. Choi, Korean J Radiol, 2008, 9, 291-302.

12 Y.-F. Zhou, World J. Clin. Oncol., 2011, 2, 8-27.

13 J. Vidal-Jove, E. Perich and M. A. del Castillo, Ultrason. Sonochem., 2015, 27, 703-706.

14 J.-F. Aubry, K. B. Pauly, C. Moonen, G. T. Haar, M. Ries, R. Salomir, S. Sokka, K. M. Sekins, Y. Shapira, F. Ye, H. HuffSimonin, M. Eames, A. Hananel, N. Kassell, A. Napoli, J. H. Hwang, F. Wu, L. Zhang, A. Melzer, Y.-S. Kim and W. M. Gedroyc, J. Ther. Ultrasound, 2013, 1, 13.

15 H. Grüll and S. Langereis, J. Contr. Release, 2012, 161, 317327.

16 P. C. Lyon, L. F. Griffiths, J. Lee, D. Chung, R. Carlisle, F. Wu, M. R. Middleton, F. V. G. and C. C. Coussios, J. Ther. Ultrasound, 2017, 5, 28.

17 S. Jauhari, S. Singh and A. K. Dash, in S. Jauhari, S. Singh and A. K. Dash, ed. H. G. Brittain, Academic Press, 2009, vol. 34 of Profiles of Drug Substances, Excipients and Related Methodology, pp. 299-344.

18 B. A. Weaver, Mol. Biol. Cell, 2014, 25, 2677-2681.

19 P. Ma and R. J. Mumper, J. Nanomed. Nanotechnol., 2013, 4, year.

20 M. C. Cochran, J. Eisenbrey, R. O. Ouma, M. Soulen and M. A. Wheatley, Int. J. Pharm., 2011, 414, 161-170.

21 M. S. Tartis, J. McCallan, A. F. Lum, R. LaBell, S. M. Stieger, T. O. Matsunaga and K. W. Ferrara, Ultrasound Med. Biol., 2006, 32, 1771-1780.

22 N. Wallace and S. P. Wrenn, Ultrasonics, 2015, 63, 31-38.

23 J. Y. Lee, D. Carugo, C. Crake, J. Owen, M. de Saint Victor,
A. Seth, C. Coussios and E. Stride, Adv. Mat., 2015, 27, 54845492.

24 N. Rapoport, A. M. Kennedy, J. E. Shea, C. L. Scaife and K.-H. Nam, Mol. Pharm., 2010, 7, 22-31.

25 B. Howard, Z. Gao, S. W. Lee, M.-H. Seo and N. Rapoport, Am. J. Drug Deliv., 2006, 4, 97-104.

26 S. Desgranges, W. Urbach, L. Somaglino, N. Taulier and C. Pepin, 2016.

27 A. G. Mailer, P. S. Clegg and P. N. Pusey, J. Phys. Condens. Matter, 2015, 27, 145102.

28 L. Somaglino, G. Bouchoux, J.-L. Mestas and C. Lafon, Ultrason. Sonochem., 2011, 18, 577-588.

29 Y. Iida, K. Yasui, T. Tuziuti and M. Sivakumar, Microchem. J., 2005, 80, 159-164.

30 T. J. Mason, J. P. Lorimer, D. M. Bates and Y. Zhao, Ultrason. Sonochem., 1994, 1, S91-S95.

31 G. J. Price, F. A. Duck, M. Digby, W. Holland and T. Berryman, Ultrason. Sonochem., 1997, 4, 165-171.

32 K. Astafyeva, L. Somaglino, S. Desgranges, R. Berti, C. Patinote, D. Langevin, F. Lazeyras, R. Salomir, A. Polidori, C. Contino-Pépin, W. Urbach and N. Taulier, J. Mater. Chem. B, 2015, 3, 2892-2907.

33 O. Lorton, J.-N. Hyacinthe, S. Desgranges, L. Gui, A. Klauser, Z. Celicanin, L. A. Crowe, F. Lazeyras, E. Allémann, N. Taulier, C. Contino-Pépin and R. Salomir, J. Magn. Reson., 2018, 295, 27-37.

34 J. G. Riess, Chem. Rev., 2001, 101, 2797-2919.

35 W. Johnson, Int. J. Toxicol., 2002, 21 Suppl 2, 1-17.

36 H. Kim, M. S. Choi, Y. S. Ji, I. S. Kim, G. B. Kim, I. Y. Bae, M. C. Gye and H. H. Yoo, Pharmaceutics, 2018, 10, year.

37 S. Jasseron, C. Contino-Pépin, J. C. Maurizis, M. Rapp and B. Pucci, Bioorg. Med. Chem. Lett., 2002, 12, 1067-1070.

38 S. Jasseron, C. Contino-Pépin, J. Maurizis, M. Rapp and B. Pucci, Eur. J. Med. Chem., 2003, 38, 825-836.

39 R. E. Apfel and C. K. Holland, Ultrasound Med. Biol., 1991, $17,179-185$.

40 J. Xuan, O. Boissière, Y. Zhao, B. Yan, L. Tremblay, S. Lacelle, H. Xia and Y. Zhao, Langmuir, 2012, 28, 16463-16468.

41 B. Taghizadeh, S. Taranejoo, S. A. Monemian, Z. S. Moghaddam, K. Daliri, H. Derakhshankhah and Z. Derakhshani, Drug Deliv., 2015, 22, 145-155.

42 P. Zarrintaj, M. Jouyandeh, M. R. Ganjali, B. S. Hadavand, M. Mozafari, S. S. Sheiko, M. Vatankhah-Varnoosfaderani, T. J. Gutiérrez and M. R. Saeb, Eur. Polymer J., 2019, 117, 402-423.

43 M. Afadzi, C. de L. Davies, Y. H. Hansen, T. Johansen, Ø. K. Standal, R. Hansen, S.-E. Måsøy, E. A. Nilssen and B. Angelsen, Ultrasound in Med. \& Biol, 2012, 38, 476-486.

44 J.-L. Mestas, R. A. Fowler, T. J. Evjen, L. Somaglino, A. Moussatov, J. Ngo, S. Chesnais, S. Røgnvaldsson, S. L. Fossheim, E. A. Nilssen and C. Lafon, J. Drug Target., 2014, 22, 688697.

45 J. Kost, K. Leong and R. Langer, Proc. Natl. Acad. Sci. U.S.A., 1989, 86, 7663-7666. 
46 E. C. Unger, T. P. McCreery, R. H. Sweitzer, V. E. Caldwell and Y. Wu, Invest. Radiol., 1998, 33, 886-892.

47 S. Paul, R. Nahire, S. Mallik and K. Sarkar, Comput. Mech., 2014, 53, 413-435.

48 Y. Zhou, J. Ther. Ultrasound, 2015, 3, 20.

49 O. Couture, M. Faivre, N. Pannacci, A. Babataheri, V. Servois, P. Tabeling and M. Tanter, Med. Phys., 2011, 38, 1116-1123.

50 C. Oerlemans, R. Deckers, G. Storm, W. E. Hennink and J. F. W. Nijsen, J. Control. Rel., 2013, 168, 327-333.

51 I. Lentacker, I. D. Cock, R. Deckers, S. C. D. Smedt and C. T. W. Moonen, Adv. Drug Deliv. Rev., 2014, 72, 49-64.

52 S. Meairs and A. Alonso, Progr. Biophys. Mol. Biol., 2007, 93, 354-362.

53 C. C. Chen, P. S. Sheeran, S.-Y. Wu, O. O. Olumolade, P. A. Dayton and E. E. Konofagou, J. Contr. Release, 2013, 172,
795-804.

54 I. V. Larina, B. M. Evers, T. V. Ashitkov, C. Bartels, K. V. Larin and R. O. Esenaliev, Technol. Cancer Res. Treat., 2005, 4, 217226.

55 G. Canavese, A. Ancona, L. Racca, M. Canta, B. Dumontel, F. Barbaresco, T. Limongi and V. Cauda, Chem. Eng. J., 2018, 340, 155-172.

56 P. Qin, T. Han, A. C. Yu and L. Xu, J. Contr. Release, 2018, 272, 169-181.

57 A. L. Y. Kee and B. M. Teo, Ultrason. Sonochem., 2019, 56, 37-45.

58 R. M. Straubinger, in R. M. Straubinger, ed. M. Suffness, CRC Press, 1995.

59 S. Eggen, M. Afadzi, E. A. Nilssen, S. B. Haugstad, B. Angelsen and C. de L. Davies, Ultrasound Med. Biol., 2013, 39, 12551266. 